\title{
Vitamin D Perspective in Front Line Healthcare Workers Amid COVID-19
}

\author{
Muhammad Waris Farooka, Syeda Saba Aslam, Mansoor Ahmed Tarar, Minhaj Rafi, Afaq Ahmad, \\ Nouman Rafique, and Muhammad Bilal
}

\section{ABSTRACT}

Deficiency of Vitamin D is very common in Pakistan, even among healthy asymptomatic individuals [1], [2]. Recent studies have shown that the risk of contracting COVID-19 was increased to two-fold, and consequent mortality to 4-fold if the person is Vitamin D deficient [3]. Health care workers including the nursing and administration staff are at a high risk of contracting SARS-CoV2 due to increased regular exposure in a health care setting [4]. Consequently, a convergence of the COVID-19 pandemic, the deficiency of Vitamin $D$, and the increased exposure can render the health care workers at an additional risk to COVID-19 infection. Our objective was to determine the prevalence of vitamin $D$ deficiency in healthy asymptomatic front-line health care workers and to analyze the change in serum level by loading oral dose of SunnyD STAT softgel capsules (200000 IU Vitamin D3). We followed single centered, cross-sectional, cohort study with subsequent randomized placebo-controlled design for supplementation and follow up. Serum level of 25-hydroxyvitamin D (25OHD) was the main outcome variable, with anthropometric data, nutritional intake, and lifestyle variables analyzed for potential association as risk factors for the outcome. Severe Vitamin D deficiency was found to be prevalent among front line health care workers in this urban hospital-based sample. Serum level of Vitamin D was found to be significantly associated with designation and presence of high blood pressure. The likelihood of increased serum Vitamin D levels was observed with increasing monthly income, higher designation, increasing age and supplementation intake. Mean increase in the serum 25(OH)D3 level after 2 doses of SunnyD STAT softgel capsule (200000 IU Vitamin D3) was $34.22 \mathrm{ng} / \mathrm{ml}$. Public health interventions regarding Vitamin D supplementation and awareness are needed, especially amid COVID-19 pandemic.

Keywords: COVID-19, healthcare workers, Vitamin D deficiency.
Submitted : Jun 30, 2021

Published : July 21, 2021

ISSN: $2593-8339$

DOI: $10.24018 /$ ejmed.2021.3.4.957

Prof. Dr. Muhammad Waris Farooka Services Institute of Medical Sciences, Lahore, Pakistan.

(e-mail: warisfarooka@gmail.com)

Dr. Syeda Saba Aslam*

Scotmann Pharmaceuticals, Pakistan.

(e-mail: medicalaffairs@ ${ }^{@}$ scotmann.com.pk) Dr. Mansoor Ahmed Tarar

Services Institute of Medical Sciences, Lahore, Pakistan.

(e-mail: mansoortarar@gmail.com)

Dr. Minhaj Rafi

Services Institute of Medical Sciences, Lahore, Pakistan.

(e-mail: minhaaj_rafi_1@ yahoo.com)

Dr. Afaq Ahmad

Services Institute of Medical Sciences, Lahore, Pakistan.

(e-mail: warisfarooka@gmail.com)

Dr. Nouman Rafique

Services Institute of Medical Sciences, Lahore, Pakistan.

(e-mail: m.noumanrafique@gmail.com)

Dr. Muhammad Bilal

Services Institute of Medical Sciences, Lahore, Pakistan.

(e-mail: m.bilalminhas002@gmail.com)

*Corresponding Author

\section{INTRODUCTION}

Vitamin D is a secosteroid that has a wide spectrum of immunomodulatory, anti-inflammatory, antifibrotic, and antioxidant actions [5]. Epidemiological studies have reported that vitamin $\mathrm{D}$ deficiency is associated with viral respiratory tract infections and acute lung injury [5]. Vitamin D supplementation has exhibited protective effects against acute lung injury by modulating the expression of members of the renin-angiotensin system such as ACE2 in lung tissue [7], supporting the role of vitamin D deficiency as a pathogenic factor in COVID-19. Type-II pneumocytes are the primary target of coronaviruses and ACE2 receptors are highly expressed on these cells [9]. Impaired function of type-
II pneumocytes decrease the surfactant level and increase surface tension in COVID-19 [9]. Metabolites of 1,25dihydroxyvitamin $\mathrm{D}$ have been reported to stimulate surfactant synthesis in alveolar type-II cells [10]. Recent studies have shown that the risk of contracting COVID-19 was increased to two-fold, and consequent mortality to 4-fold if the person is Vitamin D deficient [12]. Moreover, optimal levels of Vitamin D in the blood can reduce the risk of ICU admission to up to $97 \%$ [13].

Unfortunately, there is a high prevalence of vitamin D deficiency/insufficiency globally, in a very diverse population around the world [14]-[16]. Different studies done in Pakistan also identify this prevalence to be between 78 97\% in Pakistani population [1], [2], [17], [20]-[25]. 
Amid the current pandemic of COVID-19, the health care workers including the nursing staff and administration are at high risk of contracting SARS-CoV2 [4]. With the Vitamin $\mathrm{D}$ deficiency already endemic in the society [21], a convergence of the COVID-19 pandemic, the deficiency of Vitamin D, and the increased exposure can render the health care workers at an additional risk to COVID-19 infection. To the best of our knowledge, no study had been done to evaluate the prevalence of Vitamin D deficiency in this particular cohort. This study, therefore, aimed to cover this gap in literature by reporting the prevalence of hypovitaminosis D in this cohort, identifying the potential risk factors and to assess the change in baseline levels of Vitamin D after loading dose of supplementation, so that policy recommendations can be given for adequate public health interventions.

\section{METHODS}

\section{A. Study Population}

We enrolled 256 healthy volunteer front line health care workers as participants for this study. The front-line healthcare workers included all the working doctors, nursing staff, paramedical, administrative, and cleaning staff of the ward who had regular exposure to patients coming to the hospital and did not have any apparent signs and symptoms of active COVID-19 infection. Sample size of 256 participants was calculated according to the formula:

$$
\mathrm{n}=\mathrm{N} \times \mathrm{X} /(\mathrm{X}+\mathrm{N}-1)
$$

where

$\mathrm{X}=\mathrm{Z} \alpha / 22 \neg * \mathrm{p} *(1-\mathrm{p}) / \mathrm{MOE} 2$;

$\mathrm{Z} \alpha / 2=$ critical value of the Normal distribution at $\alpha / 2$ (confidence level of $95 \%, \alpha$ is 0.05 and the critical value is 1.96);

MOE = margin of error;

$\mathrm{p}=$ sample proportion;

$\mathrm{N}=$ population size;

Selected margin of error $=5 \%$;

Selected Confidence level $=95 \%$;

Selected Population size $=1000000$;

Sample proportion $=80 \%$ (previous studies [17], [21]).

All health care workers of the ward including doctors, nurses and admin staff were offered to be included in the trial after taking informed consent, until the desired sample of 256 was achieved.

\section{B. Consent and Ethical Approval}

Consent was obtained by receiving signature or thumb impression of the participant on the consent form. Participants were informed that the dose would be given according to their current serum levels after laboratory testing, in compliance with international guidelines [23], to avoid risk of any toxicity or adverse event. Participants were also informed that, in case of deficiency, they had equal chances of being randomly allotted to either the placebo or the supplementation group. The Institutional Review Board of SIMS hospital approved the protocol.

\section{Data Collection}

Once enrolled, the participants filled a questionnaire about their current dietary habits and lifestyle. Moreover, a brief medical, surgical and drug history was also be taken to evaluate any association with serum Vitamin D levels.

\section{Laboratory Measurements}

After filling up of the Questionnaire, participants underwent baseline screening of their serum Vitamin D status through $15 \mathrm{~mL}$ blood test sampling of serum 25(OH)D3. All tests were performed by the Chughtai lab, SIMS hospital. Serum 25(OH)D levels were measured by competitive binding assay. Lab results were shared with participants and analysts.

\section{E. Statistical Analysis}

A sample size of 256 participants was calculated using the formula specified above, with 95\% confidence level, $\alpha$ value of 0.05 and the critical value at 1.96. Descriptive data is expressed in frequencies and percentages while serum $25(\mathrm{OH}) \mathrm{D} 3$ levels are also expressed through mean, median and mode.

Data was categorized for statistical analysis. For dietary variables including egg, milk, and fish consumption, "zero consumption" was considered if the participant reported to have never consumed these foods or very rarely (hardly once a month) consumed them. Moreover, for egg consumption, those who consumed an egg daily, or 3-10 times per week were categorized as "frequent eaters" of egg. While "heavy eaters" included consumers of 10 or more eggs per week. Similarly, for milk consumption, 1-2 glasses per week were categorized as "seldom consumers", while "frequent consumption" referred to 1-2 or >2 glasses of milk every day. For fish intake, "good consumers" referred to consumption of at least 1-2 or $>2$ fish per week, or at least 1 fish daily.

For sun exposure, duration was labelled to be "no sun exposure" if the participant has no daily sun exposure or hardly 20 minutes a week. "Regular but low sun exposure" referred to those having 1-10 minutes of sun exposure every day, while 10-20 minutes or $>20$ minutes of daily sun exposure was considered "regular and good exposure".

Statistical analyses were conducted with a commercially available software program (SPSS for Windows; Version 25).

Skewed distribution was log transformed for analysis, to prevent undue influence of extreme values. $40 \mathrm{ng} / \mathrm{ml}$ or greater was considered optimal level for immune health and values at $39.9 \mathrm{ng} / \mathrm{ml}$ or below were considered sub optimal for immune protection for COVID-19 [26]. Prevalence of hypovitaminosis D in subcategories was further displayed in frequencies and percentages.

To further assess simple bivariate associations among serum $25(\mathrm{OH}) \mathrm{D}$ level, and predictor variables, we used the $\chi 2$ statistic, likelihood ratio, Fisher exact test, Pearson, and Spearman correlation coefficient as appropriate to the nature and distribution of the variables.

Pearson Chi square statistic was used for categorical (nominal) variables assuming the null hypothesis that no relationship exists between the serum 25(OH)D3 levels and the categorical variables in the population, i.e., they are independent. Cross tabulations (bivariate tables) were used to evaluate tests of independence. Continuity correction was 
applied to bivariate variables like gender, designation, taking regular medicines, presence/absence of co morbidities like diabetes, cardiovascular disease, high blood pressure, disability, and chronic body aches and pains to approximate the discrete distribution. $\mathrm{P}<0.05$ was considered to be statistically significant.

Likelihood of having below optimal levels of serum Vitamin D3 was analyzed through ratio ( $>10$ considered high) with a value of $\mathrm{p}<0.05$ considered statistically significant. Fisher exact test was also used for bivariate variables including gender and presence/absence of co morbidities including diabetes, CVD, HBP, physical disability, chronic body aches and pains. The variables went further linear to linear comparison with the serum 25(OH)D3 levels.

To assess the correlation between the interval and ordinal variables and the serum $25(\mathrm{OH}) \mathrm{D}$ level, the strength of linear relationship was analyzed using the Pearson and Spearman correlation coefficients. These included age, weight, height, monthly income, length of sun exposure and consumption of egg, milk, fish, and dietary supplements. Pearson's R was calculated for interval variables and serum Vitamin D levels, including age weight and height, based on normal approximation. Spearman's correlation was established for ordinal variables including monthly income, length of sun exposure and intake of egg, milk, fish, and dietary supplements.

\section{RESULTS}

The final sample was composed of 256 participants. After cleaning of data and removal of missing data, 199 remained valid for analysis. Baseline descriptive characteristics of the participants is given in Table I.

\section{A. Baseline Characteristics of the Participants}

The mean age of the participants was 35 years (Range 2073 years). Majority of the participants were in the younger age group with $89(44.7 \%)$ participants falling between $20-29$ years of age, $42(21.1 \%)$ in the 30-39 years age bracket, 34 $(17.1 \%)$ in the $40-49$ years age bracket and just $28(14.1 \%)$ above 50 years of age. $109(54.8 \%)$ participants were male while $90(45.2 \%)$ were females. In designations, the highest number was that of House officers $39(19.6 \%)$ of the total sample, followed by nurses comprising of $11.1 \%(n=22)$ of the total sample. $46(23.1 \%)$ participants were earning in the salary range between Rs. 20000-50000/month, while just 20 (10.1\%) participants had a salary above Rs.100000/month.

$71.9 \%(\mathrm{n}=143)$ participants had weight above $50 \mathrm{~kg}$, while $23.6 \%(n=47)$ had weight between $41-50 \mathrm{~kg}$. Presence of co morbidities including diabetes, cardiovascular diseases, high blood pressure and chronic body aches and pains was $7.5 \%$ $(\mathrm{n}=15), \quad 3 \% \quad(\mathrm{n}=6), 6.5 \% \quad(\mathrm{n}=13) \quad$ and $12.6 \% \quad(\mathrm{n}=25)$ respectively.

$35.2 \%(\mathrm{n}=70)$ participants had nil to minimal sun exposure (hardly 20 minutes/week), 21.1\% (n=42) had regular but low sun exposure while $24.6 \%(n=49)$ had regular and good sun exposure (See Methods for definitions of categories).

$58.8 \%(n=117)$ participants were frequent eaters of egg on a daily basis, while $17.1 \%(n=34)$ seldom or rarely ate eggs. $13.1 \%(n=26)$ ate 10 or more eggs per week and were categorized as heavy eaters of egg. $44.7 \%(\mathrm{n}=89)$ almost never drink milk, while $38.7 \%(n=77)$ consumed only $1-2$ glasses per week. Only $13.1 \%(n=26)$ of the participants regularly drank at least 1 or 2 glasses of milk. Fish consumption was very scarce with $85.9 \%(n=171)$ of the participants hardly eating 1 or 2 fish in a month, while only $11.1 \%(n=22)$ reported to consume at least $1-2$ fish per week or daily.

\begin{tabular}{|c|c|}
\hline Characteristics & Value n (\%) \\
\hline \multicolumn{2}{|l|}{ Age $y$. valid $=193$ missing $=6$} \\
\hline Mean +-SD & $35.09+-10.79$ \\
\hline Range & $20-73$ \\
\hline $20-29$ & $89(44.7 \%)$ \\
\hline $30-39$ & $42(21.1 \%)$ \\
\hline $40-49$ & $34(17.1 \%)$ \\
\hline $50-59$ & $26(13.1 \%)$ \\
\hline$>60$ & $2(1 \%)$ \\
\hline \multicolumn{2}{|l|}{ Gender Valid $=199$ missing $=0$} \\
\hline Male & $109(54.8 \%)$ \\
\hline Female & $90(45.2 \%)$ \\
\hline \multicolumn{2}{|c|}{ Designation Valid $=188$ missing $=11$} \\
\hline Medical Student & $19(9.5 \%)$ \\
\hline House officer & $39(19.6 \%)$ \\
\hline Resident & $20(10.1 \%)$ \\
\hline Assistant Professor & $3(1.5 \%)$ \\
\hline Professor & $4(2 \%)$ \\
\hline Head of department & $3(1.5 \%)$ \\
\hline Consultant & $5(2.5 \%)$ \\
\hline Nurse & $22(11.1 \%)$ \\
\hline Head Nurse & $4(2 \%)$ \\
\hline Administration Staff & $2(1 \%)$ \\
\hline Nursing Superintendent & $8(4 \%)$ \\
\hline Other & $59(29.6 \%)$ \\
\hline \multicolumn{2}{|c|}{ Monthly income Valid $=156$ missing $=43$} \\
\hline $5000-20000$ & $23(11.6 \%)$ \\
\hline $20000-50000$ & $46(23.1 \%)$ \\
\hline $50000-100000$ & $34(17.1 \%)$ \\
\hline Above Rs. 100000 & $20(10.1 \%)$ \\
\hline Other & $33(16.6 \%)$ \\
\hline \multicolumn{2}{|c|}{ Serum D3 levels total $=160$, missing $=39$} \\
\hline Below optimal* & $118(59.3 \%)$ \\
\hline optimal* & $42(21.1 \%)$ \\
\hline Mean +- SD & $\begin{array}{l}32.03+- \\
32.801\end{array}$ \\
\hline Median & 18.95 \\
\hline Mode & 7.4 \\
\hline Range & $5.6-154.2$ \\
\hline \multicolumn{2}{|l|}{ Weight valid $=198$ missing $=1$} \\
\hline $21-30$ & $1(0.5 \%)$ \\
\hline $31-40$ & $1(0.5 \%)$ \\
\hline $41-50$ & $6(3 \%)$ \\
\hline $51-60$ & $47(23.6 \%)$ \\
\hline Above 60 & $143(71.9 \%)$ \\
\hline \multicolumn{2}{|l|}{ Diabetes valid $=197$ missing $=2$} \\
\hline No & $182(91.5 \%)$ \\
\hline Yes & $15(7.5 \%)$ \\
\hline \multicolumn{2}{|l|}{ CVD valid $=196$ missing $=3$} \\
\hline No & $190(95.5 \%)$ \\
\hline Yes & $6(3 \%)$ \\
\hline \multicolumn{2}{|l|}{ HBP valid $=196$ missing $=3$} \\
\hline No & $183(92 \%)$ \\
\hline Yes & $13(6.5 \%)$ \\
\hline \multicolumn{2}{|c|}{ Any other Illness/disability valid $=159$ missing $=40$} \\
\hline No & $146(73.4 \%)$ \\
\hline Yes & $1396.5 \%)$ \\
\hline \multicolumn{2}{|c|}{ Egg consumption valid $=177$ missing $=22$} \\
\hline Zero eaters & $34(17.1 \%)$ \\
\hline Frequent eaters & $117(58.8 \%)$ \\
\hline Heavy eaters & $26(13.1 \%)$ \\
\hline \multicolumn{2}{|c|}{ Milk Intake valid $=192$ missing $=7$} \\
\hline Zero & $89(44.7 \%)$ \\
\hline seldom & $77(38.7 \%)$ \\
\hline
\end{tabular}


TABLE I: CONTD.

\begin{tabular}{ll}
\hline \hline \multicolumn{1}{c}{ Characteristics } & \multicolumn{1}{c}{ Value n (\%) } \\
\hline Frequent & $26(13.1 \%)$ \\
Fish intake valid= 193, missing=6 & $171(85.9 \%)$ \\
Nill to rare & $22(11.1 \%)$ \\
Good consumers & \\
Medicines valid=160 missing =39 & $159(99.5 \%)$ \\
No & $1(0.5 \%)$ \\
Yes & \\
Chronic body aches valid = 171 missing $=28$ & $146(73.4 \%)$ \\
No & $25(12.6 \%)$ \\
Yes & $135(67.8 \%)$ \\
Vitamin D supplements intake valid=187 missing=12 & $25(12.6 \%)$ \\
No. I don't take any supplement & \\
I take supplements but I don't & \\
remember the amount of Vitamin D in & \\
it & $5(2.5 \%)$ \\
0-500 IU per day & $1(0.5 \%)$ \\
$0-500$ IU per week & $4(2 \%)$ \\
500-1000 IU per day & $3(1.5 \%)$ \\
5000-10000 IU per day & $12(6 \%)$ \\
200000 IU once a month & \\
Sun exposure valid=161 missing = 38 & $70(35.2 \%)$ \\
No sun exposure & $42(21.1 \%)$ \\
Regular but low sum exposure & $49(24.6 \%)$ \\
Regular and good exposure & \\
\hline \hline
\end{tabular}

$*>40 \overline{\mathrm{ng} / \mathrm{ml}}$.

$* *<40 \mathrm{ng} / \mathrm{ml}$.

\section{B. Prevalence of hypovitaminosis $D$}

The prevalence of sub optimal levels of Vitamin D3 in the total sample was $74.8 \%$; with severe deficiency $(<10 \mathrm{ng} / \mathrm{ml})$ observed in $26.7 \%(n=42)$ of the participants. The lowest value reaching at $5.6 \mathrm{ng} / \mathrm{ml}$, and a mode of $7.4 \mathrm{ng} / \mathrm{ml}$ was observed. The mean serum 25(OH)D3 was $32.03 \mathrm{ng} / \mathrm{ml}$ and a median serum 25(OH)D3 level of $18.95 \mathrm{ng} / \mathrm{ml}$ was calculated in the sample. The prevalence of hypovitaminosis within subgroups is listed in Table III, with the highest prevalence observed in young age participants between 20-29 years of age with $93.4 \%$ of them being deficient, zero eaters of egg $(82.41 \%$ deficient), no supplements intake $(88.2 \%$ deficient) and with nil to less than 20 minutes per week sun exposure (75.53\% deficient).

The serum 25OHD level showed a skewed distribution and was accordingly $\log$ transformed for analysis, to prevent undue influence of extreme values.

TABLE II: PREVALENCE OF HYPOVITAMINOSIS D IN SUBGROUPS FREQUENCY TABLE

\begin{tabular}{lccc}
\hline \hline Characteristics & $\begin{array}{c}\text { Optimal*n } \\
(\%)\end{array}$ & $\begin{array}{c}\text { Below } \\
\text { optimal** } \mathrm{n}(\%)\end{array}$ & Total \\
\hline Age y. & $5(6.5 \%)$ & $71(93.4 \%)$ & 76 \\
$20-29$ & $12(37.5 \%)$ & $20(62.5 \%)$ & 32 \\
$30-39$ & $12(48 \%)$ & $13(52 \%)$ & 25 \\
$40-49$ & $11(47.82 \%)$ & $12(52.17 \%)$ & 23 \\
$50-59$ & $1(100 \%)$ & $0(0 \%)$ & 1 \\
$>60$ & & & \\
Gender & $20(23.25 \%)$ & $66(76.7 \%)$ & 86 \\
Male & $22(29.7 \%)$ & $52(70.27 \%)$ & 74 \\
Female & & & \\
Designation & $2(12.5 \%)$ & $14(87.5 \%)$ & 16 \\
Medical Student & $3(0.08 \%)$ & $31(91.17 \%)$ & 34 \\
House officer & $2(12.5 \%)$ & $14(87.5 \%)$ & 16 \\
Resident & $0(0 \%)$ & $3(100 \%)$ & 3 \\
Assistant Professor & $1(25 \%)$ & $3(75 \%)$ & 4 \\
Professor & $3(100 \%)$ & $0(0 \%)$ & 3 \\
Head of department & $1(25 \%)$ & $3(75 \%)$ & 4 \\
Consultant & $6(33.3 \%)$ & $12(66.66 \%)$ & 18 \\
Nurse & $2(66.6 \%)$ & $1(33.34 \%)$ & 3 \\
Head Nurse & $1(50 \%)$ & $1(50 \%)$ & 2 \\
Administration Staff & & &
\end{tabular}

\begin{tabular}{|c|c|c|c|}
\hline $\begin{array}{l}\text { Nursing } \\
\text { Superintendent }\end{array}$ & $7(87.5 \%)$ & $1(12.5 \%)$ & 8 \\
\hline Other & $12(30 \%)$ & $28(70 \%)$ & 40 \\
\hline \multicolumn{4}{|l|}{ Monthly income } \\
\hline $5000-20000$ & $3(16.67 \%)$ & $15(83.3 \%)$ & 18 \\
\hline $20000-50000$ & $9(25.71 \%)$ & $26(74.29 \%)$ & 35 \\
\hline $50000-100000$ & $10(35.71 \%)$ & $18(64.29 \%)$ & 28 \\
\hline Above Rs. 100000 & $11(57.89 \%)$ & $8(42.11 \%)$ & 19 \\
\hline Other & $2(7.14 \%)$ & $26(92.8 \%)$ & 28 \\
\hline \multicolumn{4}{|l|}{ Weight } \\
\hline $21-30$ & $0(0 \%)$ & $1(100 \%)$ & 1 \\
\hline $31-40$ & $0(0 \%)$ & $1(100 \%)$ & 1 \\
\hline $41-50$ & $1(25 \%)$ & $3(75 \%)$ & 4 \\
\hline $51-60$ & $9(21.9 \%)$ & $32(78.04 \%)$ & 41 \\
\hline Above 60 & $32(28.31 \%)$ & $81(71.68 \%)$ & 113 \\
\hline \multicolumn{4}{|l|}{ Diabetes } \\
\hline No & $36(24.65 \%)$ & $110(75.34 \%)$ & 146 \\
\hline Yes & $\begin{array}{c}5(41.66 \%) \\
\text { CVD }\end{array}$ & $7(58.3 \%)$ & 12 \\
\hline No & $38(24.6 \%)$ & $116(75.3 \%)$ & 154 \\
\hline Yes & $2(66.6 \%)$ & $1(33.3 \%)$ & 3 \\
\hline \multicolumn{4}{|l|}{ HBP } \\
\hline No & $34(22.97 \%)$ & $114(77 \%)$ & 148 \\
\hline Yes & $6(66.6 \%)$ & $3(33.3 \%)$ & 9 \\
\hline \multicolumn{4}{|l|}{ Any other Illness/disability } \\
\hline No & $32(27.1 \%)$ & $86(72.8 \%)$ & 118 \\
\hline Yes & $1(10 \%)$ & $9(90 \%)$ & 10 \\
\hline \multicolumn{4}{|l|}{ Egg consumption } \\
\hline Zero eaters & $5(17.85 \%)$ & $23(82.14 \%)$ & 28 \\
\hline Frequent eaters & $23(24.46 \%)$ & $71(75.53 \%)$ & 94 \\
\hline Heavy eaters & $9(41 \%)$ & $13(59 \%)$ & 22 \\
\hline \multicolumn{4}{|l|}{ Milk Intake } \\
\hline Zero & $19(26 \%)$ & $54(73.97 \%)$ & 73 \\
\hline seldom & $16(27.58 \%)$ & $42(72.41 \%)$ & 58 \\
\hline Frequent & $4(17.4 \%)$ & $19(82.6 \%)$ & 23 \\
\hline & Fish intake & & \\
\hline Nill to rare & $34(25.18 \%)$ & $101(74.81 \%)$ & 135 \\
\hline Good consumers & $6(30 \%)$ & $14(70 \%)$ & 20 \\
\hline \multicolumn{4}{|l|}{ Chronic body aches } \\
\hline No & $29(24.7 \%)$ & $88(75.2 \%)$ & 117 \\
\hline Yes & $7(31.8 \%)$ & $15(68.18 \%)$ & 22 \\
\hline \multicolumn{4}{|c|}{ Vitamin D supplements intake } \\
\hline $\begin{array}{l}\text { No. I do not take any } \\
\text { supplement }\end{array}$ & $13(11.7 \%)$ & $98(88.2 \%)$ & 11 \\
\hline $\begin{array}{l}\text { I take supplements } \\
\text { but I do not }\end{array}$ & & & \\
\hline $\begin{array}{l}\text { remember the } \\
\text { amount of Vitamin } \\
D \text { in it }\end{array}$ & $9(56.25 \%)$ & $7(43.75 \%)$ & 16 \\
\hline 0-500 IU per day & $1(20 \%)$ & $4(80 \%)$ & 5 \\
\hline $500-1000$ IU per day & $1(25 \%)$ & $3(75 \%)$ & 4 \\
\hline $\begin{array}{l}5000-10000 \mathrm{IU} \text { per } \\
\text { day }\end{array}$ & $3(100 \%)$ & $0(0 \%)$ & 3 \\
\hline $\begin{array}{l}200000 \text { IU once a } \\
\text { month }\end{array}$ & $7(63.63 \%)$ & $4(36.36 \%)$ & 11 \\
\hline \multicolumn{4}{|l|}{ Sun exposure } \\
\hline No sun exposure & $14(24.56 \%)$ & $43(75.53 \%)$ & 57 \\
\hline $\begin{array}{l}\text { Regular but low sum } \\
\text { exposure }\end{array}$ & $7(20 \%)$ & $28(80 \%)$ & 35 \\
\hline $\begin{array}{l}\text { Regular and good } \\
\text { exposure }\end{array}$ & $14(34.14 \%)$ & $27(65.85 \%)$ & 41 \\
\hline
\end{tabular}

*Optimal $=40 \mathrm{ng} / \mathrm{ml}$ and above.

$* *$ Below optimal $=39.9 \mathrm{ng} / \mathrm{ml}$ and below.

\section{Variables Associated with Low 25(OH)D3 Levels}

Among the categorical (nominal) variables, the levels of serum $25(\mathrm{OH}) \mathrm{D} 3$ did not differ by gender $(\mathrm{X} 2=0.861$ $\mathrm{p}=0.353)$, presence/absence of co morbidities like diabetes $(\mathrm{X} 2=1.669 \mathrm{p}=0.196)$, cardiovascular disease $(\mathrm{X} 2=2.733 \mathrm{p}=$ $0.098)$, any physical disability $(\mathrm{X} 2=1.412 \mathrm{p}=0.235)$ and chronic body aches and pains $(\mathrm{X} 2=0.477 \mathrm{p}=0.49)$. However, designation $(\mathrm{X} 2=37.113 \mathrm{p}=0.000)$ and high blood pressure (X2=8.531 $\mathrm{p}=0.003)$, was strongly associated with serum Vitamin D levels.

The likelihood of increased serum Vitamin D levels (high 
likelihood ratio $>10$ ) was observed with increasing monthly income (likelihood=17.286 $\mathrm{p}=0.002$ ), higher designation (likelihood=36.785 $\mathrm{p}=0.000$ ), increasing age (likelihood= $34.64 \mathrm{p}=0.000$ ) and supplementation intake (likelihood = $46.406 \mathrm{p}=0.000)$.

Using Fisher Exact test, higher odds for hypovitaminosis was seen in patients having chronic body aches and pains (exact significance 2 sided $=0.596$, one sided $=0.327$ ) and those having some form of disability (exact significance 2 sided $=0.451$, one sided $=0.215)$. Designation $(12.25$ $\mathrm{p}=0.000)$, Age $(26.968 \mathrm{p}=0.000)$ and intake of supplements (32.659 $\mathrm{p}=0.000)$ showed high linear by linear association and positive correlation with serum Vitamin D3 levels.

TABLE III: CHI SQUARE TESTS

\begin{tabular}{|c|c|c|c|c|c|}
\hline Test & Value & df & $\begin{array}{l}\text { Asymptotic } \\
\text { significance } \\
(2 \text { sided }) \\
\end{array}$ & $\begin{array}{l}\text { Exact sig } \\
(2 \text { sided })\end{array}$ & $\begin{array}{l}\text { Exact sign } \\
\text { (1-sided) }\end{array}$ \\
\hline \multicolumn{6}{|c|}{ Vitamin D*Gender } \\
\hline Pearson Chi square & 0.861 (a) & 1 & 0.353 & & \\
\hline Continuity correction (b) & 0.559 & 1 & 0.455 & & \\
\hline Likelihood ratio & 0.859 & 1 & 0.354 & & \\
\hline Fisher Exact test & & & & 0.373 & 0.227 \\
\hline $\begin{array}{l}\text { Linear by linear } \\
\text { Association }\end{array}$ & 0.856 & 1 & $0(0 \%)$ & 1 & \\
\hline $\mathrm{N}$ of valid cases & 160 & & & & \\
\hline \multicolumn{6}{|c|}{ Vitamin D3 $*$ designation } \\
\hline Pearson Chi square & 37.113 (a) & 11 & 0.000 . & & \\
\hline Likelihood ratio & 36.785 & 11 & 0.000 . & & \\
\hline $\begin{array}{l}\text { Linear by linear } \\
\text { Association }\end{array}$ & 12.25 & 1 & 0.000 . & & \\
\hline $\mathrm{N}$ of valid cases & 151 & & & & \\
\hline \multicolumn{6}{|c|}{ Vitamin D3 * Diabetes } \\
\hline Pearson Chi square & 1.669 & 1 & & & \\
\hline Continuity correction (b) & 0.902 & 1 & & & \\
\hline Likelihood ratio & 1.524 & 1 & & & \\
\hline Fisher Exact Test & & & & 0.301 & 0.169 \\
\hline $\begin{array}{l}\text { Linear by linear } \\
\text { Association }\end{array}$ & 1.659 & 1 & & & \\
\hline $\mathrm{N}$ of valid cases & 158 & & & & \\
\hline \multicolumn{6}{|c|}{ Vitamin D3 $*$ CVDs } \\
\hline Pearson Chi square & 2.733 (a) & 1 & 0.098 & & \\
\hline Continuity correction (b) & & 1 & 0.325 & & \\
\hline Likelihood ratio & & 1 & 0.13 & & \\
\hline Fisher Exact Test & & & & 0.16 & 0.16 \\
\hline $\begin{array}{l}\text { Linear by linear } \\
\text { Association } \\
\mathrm{N} \text { of valid cases }\end{array}$ & & 1 & 0.099 & & \\
\hline \multicolumn{6}{|c|}{ Vitamin D3 * HBP } \\
\hline Pearson Chi square & 8.531 (a) & 1 & 0.003 & & \\
\hline Continuity correction (b) & 6.385 & 1 & 0.012 & & \\
\hline Likelihood ratio & 7.216 & 1 & 0.007 & & \\
\hline Fisher Exact Test & & & & 0.009 & 0.009 \\
\hline $\begin{array}{l}\text { Linear by linear } \\
\text { Association }\end{array}$ & 8.477 & 1 & 0.004 & & \\
\hline $\mathrm{N}$ of valid cases & 157 & & & & \\
\hline \multicolumn{6}{|c|}{ Vitamin D3 * Disability } \\
\hline Pearson Chi square & 1.412 & 1 & 0.235 & & \\
\hline Continuity correction (b) & 0.659 & 1 & 0.417 & & \\
\hline Likelihood ratio & 1.685 & 1 & 0.194 & & \\
\hline Fisher Exact Test & & & & 0.451 & 0.215 \\
\hline $\begin{array}{l}\text { Linear by linear } \\
\text { Association }\end{array}$ & 1.401 & 1 & 0.237 & & \\
\hline $\mathrm{N}$ of valid cases & 128 & & & & \\
\hline \multicolumn{6}{|c|}{ Vitamin D3 $*$ chronic body aches and pains } \\
\hline Pearson Chi square & 0.477 (a) & 1 & 0.49 & & \\
\hline Continuity correction (b) & 0.181 & 1 & 0.67 & & \\
\hline Likelihood ratio & 0.460 . & 1 & 0.497 & & \\
\hline Fisher Exact Test & & & & & 0.596 \\
\hline $\begin{array}{l}\text { Linear by linear } \\
\text { Association }\end{array}$ & 0.474 & 1 & 0.491 & & \\
\hline $\mathrm{N}$ of valid cases & 139 & & & & \\
\hline
\end{tabular}

b. Computed only for a $2 \times 2$ table.

\section{Symmetric Measures}

Based on normal approximation, statistically significant but moderately positive correlation was seen between age $(\mathrm{R}=0.416, \mathrm{p}=0.000)$ and serum $25(\mathrm{OH}) \mathrm{D} 3$ levels, through the Pearson R coefficient.
Among ordinal variables, a statistically significant and moderately positive linear correlation was observed between the intake of supplements $(\mathrm{R}=0.465, \mathrm{p}=0.000)$ and serum Vitamin D levels. Weakly positive association was seen between increasing weight $(\mathrm{R}=0.084, \mathrm{p}=0.290)$, egg consumption $(\mathrm{R}=0.150 \mathrm{p}=0.073)$, milk intake $(\mathrm{R}=0.013$ 
$\mathrm{p}=0.873)$, fish intake $(\mathrm{R}=0.037 \mathrm{p}=0.649)$, duration of sun exposure $(R=0.085 \mathrm{p}=0.333$ and serum Vitamin $\mathrm{D} 3$ levels but were all statistically non-significant $(p>0.05)$, using the Spearman's rank correlation. The results of the symmetric measures statistics are summarized in Table IV.

\section{E. Increase in Serum Vitamin D3 Levels}

Mean increase in the serum 25(OH)D3 level in the interventional group after 2 doses of SunnyD STAT softgel capsule (200000 IU Vitamin D3) was $34.22 \mathrm{ng} / \mathrm{ml}$. The mean increase in the placebo group was $3.04 \mathrm{ng} / \mathrm{ml}$.

TABLE IV: SYMMETRIC MEASURES

\begin{tabular}{|c|c|c|c|c|c|}
\hline & & Value & $\begin{array}{c}\text { Asymptotic } \\
\text { SE (a) }\end{array}$ & $\begin{array}{l}\text { Appr. T } \\
\text { (b) }\end{array}$ & Appr. sig. (c) \\
\hline \multicolumn{6}{|c|}{ Vitamin D*Monthly income } \\
\hline Interval by Interval & Pearson's R & -0.014 & 0.077 & -0.158 & $0.875(\mathrm{c})$ \\
\hline Ordinal by ordinal & $\begin{array}{c}\text { Spearman } \\
\text { Correlation }\end{array}$ & 0.000 & 0.080 . & 0.000 & $1.000 .(\mathrm{c})$ \\
\hline $\mathrm{N}$ of valid cases & & 160 & & & \\
\hline \multicolumn{6}{|c|}{ Vitamin D3 $*$ Ages } \\
\hline Interval by Interval & Pearson's R & 0.416 & 0.072 & 5.692 & $0.000(\mathrm{c})$ \\
\hline Ordinal by ordinal & $\begin{array}{l}\text { Spearman } \\
\text { Correlation }\end{array}$ & 0.435 & 0.067 & 6.007 & $0.000(\mathrm{c})$ \\
\hline $\mathrm{N}$ of valid cases & & 157 & & & \\
\hline \multicolumn{6}{|c|}{ Vitamin D3 * Weight } \\
\hline Interval by Interval & Pearson's R & 0.084 & 0.066 & 1.062 & $0.290(\mathrm{c})$ \\
\hline Ordinal by ordinal & $\begin{array}{l}\text { Spearman } \\
\text { Correlation }\end{array}$ & 0.075 & 0.075 & 0.949 & $0.344(\mathrm{c})$ \\
\hline $\mathrm{N}$ of valid cases & & 160 & & & \\
\hline \multicolumn{6}{|c|}{ Vitamin D3 $*$ Egg consumption } \\
\hline Interval by Interval & Pearson's R & 0.150 & 0.084 & 1.806 & 0.073 \\
\hline Ordinal by ordinal & $\begin{array}{c}\text { Spearman } \\
\text { Correlation }\end{array}$ & 0.149 & 0.083 & 1.792 & 0.075 \\
\hline $\mathrm{N}$ of valid cases & & 144 & & & \\
\hline \multicolumn{6}{|c|}{ Vitamin D3 $*$ Milk intake } \\
\hline Interval by Interval & Pearson's R & 0.013 & 0.082 & 0.16 & 0.873 \\
\hline Ordinal by ordinal & $\begin{array}{l}\text { Spearman } \\
\text { Correlation }\end{array}$ & 0.013 & 0.082 & 0.129 & 0.898 \\
\hline $\mathrm{N}$ of valid cases & & 154 & & & \\
\hline \multicolumn{6}{|c|}{ Vitamin D3 $*$ Fish intake } \\
\hline Interval by Interval & Pearson's R & 0.037 & 0.084 & 0.457 & 0.649 \\
\hline Ordinal by ordinal & $\begin{array}{l}\text { Spearman } \\
\text { Correlation }\end{array}$ & 0.037 & 0.084 & 0.457 & 0.649 \\
\hline $\mathrm{N}$ of valid cases & & 155 & & & \\
\hline \multicolumn{6}{|c|}{ Vitamin D*Vit D supplements } \\
\hline Interval by Interval & Pearson's R & 0.465 & 0.079 & 6.434 & 0 \\
\hline Ordinal by ordinal & $\begin{array}{c}\text { Spearman } \\
\text { Correlation }\end{array}$ & 0.515 & 0.084 & 7.352 & 0 \\
\hline $\mathrm{N}$ of valid cases & & 152 & & & \\
\hline \multicolumn{6}{|c|}{ Vitamin D*Sun exposure } \\
\hline Interval by Interval & Pearson's R & 0.085 & 0.089 & 0.972 & 0.333 \\
\hline Ordinal by ordinal & $\begin{array}{l}\text { Spearman } \\
\text { Correlation }\end{array}$ & 0.08 & 0.089 & 0.918 & 0.36 \\
\hline $\mathrm{N}$ of valid cases & & 133 & & & \\
\hline
\end{tabular}

a. Not assuming the null hypothesis.

b. Using the asymptotic standard error assuming the null hypothesis.

c. Based on normal approximation.

Apr = Approximate.

$\mathrm{SE}=$ Standard Error

\section{DISCUSSION}

To the best of our knowledge, this study was the first to analyze the serum $25(\mathrm{OH}) \mathrm{D} 3$ levels in health care workers. The cross-sectional design gave a near-to-real snapshot of the hypovitaminosis picture in this cohort. The triple blinded placebo-controlled intervention added to the reliability of the results.

We found a high prevalence of severe vitamin D deficiency among otherwise healthy, asymptomatic, working health care workers who are regularly exposed to patients from one of the main public sector hospitals of the city. These findings add to growing data of the high prevalence of hypovitaminosis among other young, healthy, asymptomatic adults of Pakistan [17], [24], [25]. Previous studies [18]-[22] highlighted the prevalence among general populations or in different cohorts of patients, but to the best of our knowledge, this is the first study done in Pakistan to highlight the deficiency faced by those who are the most vulnerable at the front line of this pandemic war. These findings suggest that the problem of Vitamin D deficiency is spanning even the spectrum of healthy adults in the working health care sector.

To our surprise, young doctors were found to be comparatively more deficient than their seniors. Despite the decreasing ability of skin to synthesize Vitamin D from sunlight with age [27], [37], the effect of co morbidities [28], [38] and uptake of medications [29], [30], this finding of the high prevalence of hypovitaminosis in the youth is further alarming.

Another interesting finding was that females were comparatively less deficient than males, contrary to the expected element of more covered clothing [27], [31], lesser 
outdoor time [32] and consequently lesser exposure to sunlight [32], [33].

Among dietary habits, low egg consumption was found to be another risk factor for low serum levels of 25(OD)D3, with statistically significant values and a high prevalence of hypovitaminosis D among participants with zero to minimal egg consumption while fish and milk intake moderately influenced the serum D3 levels, with no statistically significant values.

Another expected result was that for supplementation intake, with low prevalence of hypovitaminosis D in those taking regular Vitamin D supplements.

Low sun exposure was identified as another major risk factor for hypovitaminosis D. With availability of near to ample sunlight throughout the year in the country, the heliophobic tendency of South Asian culture [34], sun avoidance behavior [35], fear of tanning [34], indoor working hours [32], lack of deliberate sun exposure [32] and covered clothing [27], [31] could account for the high prevalence of Vitamin D deficiency in Pakistan.

All the risk factors identified above are easily modifiable. Therefore, awareness about the high prevalence of Vitamin D deficiency in Pakistan should be prioritized especially for atrisk population which is most vulnerable during this pandemic.

\section{WHAT THIS STUDY ADDS}

Contrary to most studies done in this region on the prevalence of hypovitaminosis in patients coming to health care facilities or general population, our sample was composed of those who are at greatest risk during any epidemic. To the best of our knowledge, this was the first study done on healthy, asymptomatic, health care workers of the region. The findings of such high prevalence of hypovitaminosis more than expected for this cohort. This study also contradicts the results of previous studies 36 of the higher prevalence of hypovitaminosis in females compared to males, and young compared to elderly [37], [38].

\section{LIMITATIONS}

The study was cross sectional; therefore, causality cannot be inferred. Future longitudinal studies analyzing the identified risk factors can confirm the influence of these variables with more definite interpretations. Additionally, the inherent limitations of reporting bias in a questionnaire-based data collection tool could be present. Secondly, some participants didn't answer the questionnaire completely or were lost at follow up, resulting in a decrease in the data remaining valid for final analysis. Moreover, the sample was limited to health care workers so generalizability of the results to general population should be extrapolated with caution.

\section{CONCLUSIONS}

There is a high prevalence of severe Vitamin D deficiency in front line, apparently healthy, asymptomatic health care workers in Pakistan. This study can provide grounds for public health interventions like Vitamin D supplementation and raising awareness among this cohort.

\section{REFERENCES}

[1] Iqbal, Khalida, et al. "Relationship of sociodemographic factors with serum levels of vitamin D in a healthy population of Pakistan." Pakistan journal of pharmaceutical sciences 32.1 (2019): 29.

[2] Syed, Faiza, et al. "Vitamin D deficiency in Pakistani population: critical overview from 2008 to 2018." Nutrition \& Food Science (2019).

[3] De Smet, Dieter, et al. "Serum $25(\mathrm{OH})$ D level on hospital admission associated with COVID-19 stage and mortality." American journal of clinical pathology (2020).

[4] Cubitt, Lyndsey Jayne, et al. "Beyond PPE: a mixed qualitativequantitative study capturing the wider issues affecting doctors' wellbeing during the COVID-19 pandemic." BMJ open 11.3 (2021): e050223.

[5] Hughes DA, Norton R. Vitamin D and respiratory health. Clin Exp Immunol. 2009;158:20-5.

[6] Greiller CL, Martineau AR. Modulation of the immune response to respiratory viruses by vitamin $D$. Nutrients.

[7] Xu J, Yang J, Chen J, Luo Q, Zhang Q, Zhang H. Vitamin D alleviates lipopolysaccharide induced acute lung injury via regulation of the renin angiotensin system

[8] Hansdottir S, Monick MM. Vitamin D effects on lung immunity and respiratory diseases. Vitam Horm. 2011;86:217-37.

[9] Bombardini T, Picano E. Angiotensin converting enzyme 2 as the molecular bridge between epidemiologic and clinical features of COVID-19. Can J Cardiol. 2020.

[10] Rehan VK, Torday JS, Peleg S, Gennaro L, Vouros P, Padbury J, et al. 1Alpha,25-dihydroxy-3-epi-vitamin D3, a natural metabolite of 1alpha,25-dihydroxy vitamin D3: production and biological activity studies in pulmonary alveolar type II cells. Mol Genet Metab. 2002;76:46-56.

[11] Phokela SS, Peleg S, Moya FR, Alcorn JL. Regulation of human pulmonary surfactant protein gene expression by 1alpha,25dihydroxyvitamin D3. Am J Physiol Lung Cell Mol Physiol. 2005;289:617-26.

[12] De Smet, Dieter, et al. "Serum $25(\mathrm{OH})$ D level on hospital admission associated with COVID-19 stage and mortality." American journal of clinical pathology (2020).

[13] Castillo, Marta Entrenas, et al. "Effect of calcifediol treatment and best available therapy versus best available therapy on intensive care unit admission and mortality among patients hospitalized for COVID-19: A pilot randomized clinical study." The Journal of steroid biochemistry and molecular biology 203 (2020): 105751.

[14] Holick MF. Vitamin D Deficiency. N Engl J Med 2007; 357: 266-81.

[15] Nagpal S, Na S, Rathnachalam R. Noncalcemic actions of vitamin D receptor ligands. Endocr Rev 2005; 26: 662-87.

[16] Hollick MF, Chen TC. Vitamin D deficiency: a worldwide problem with health consequences. Am J Clin Nutr 2008; 87: 1080S-6S.

[17] Riaz, H., et al. "Prevalence of Vitamin D deficiency in Pakistan and implications for the future." Expert review of clinical pharmacology 9.2 (2016): 329-338.

[18] Zuberi LM, Habib A, Haque N, Jabbar A. Vitamin D deficiency in ambulatory patients. J Pak Med Assoc 2008; 58: 482-4.

[19] Baig A, Anjum P, Khani MK, Islam N, Rahman A. Pattern of serum Vitamin D in OPD patients. Pak J Surg 2007; 23: 145-9.

[20] Rab SM. Occult osteomalacia amongst health and pregnant women in Pakistan. Lancet 1976; 2: 1211-3.

[21] Atiq M, Suria A, Nizami SQ, Ahmed I. Maternal Vitamin D deficiency in Pakistan. Acta Obstet Gynaecol Scand 1998; 77, 970-3.

[22] Sahibzada AS, Khan MS, Javed M. Presentation of osteomalacia in Kohistani women. J Ayub Med Coll Abottabad 2004; 16: 63-5.

[23] van Groningen, Lenneke, et al. "Cholecalciferol loading dose guideline for vitamin D-deficient adults." European Journal of Endocrinology 162.4 (2010): 805-811. Accessed on $12^{\text {th }}$ February 2021 via https://eje.bioscientifica.com/view/journals/eje/162/4/805.xml.

[24] Syed, Faiza, et al. "Vitamin D deficiency in Pakistani population: critical overview from 2008 to 2018." Nutrition \& Food Science (2019): Kandhro, Farhatullah, et al. "Study of Vitamin D deficiency and contributing factors in the population of Hyderabad, Pakistan." Pakistan Journal of Pharmaceutical Sciences 32.3 (2019).

[25] Iqbal, Khalida, et al. "Relationship of sociodemographic factors with serum levels of vitamin D in a healthy population of Pakistan." Pakistan journal of pharmaceutical sciences 32.1 (2019): 29. 
[26] Meltzer, David O., et al. "Association of Vitamin D Levels, Race/Ethnicity, and Clinical Characteristics With COVID-19 Test Results." JAMA network open 4.3 (2021): e214117-e214117.

[27] Wang, Li-Kai, et al. "Age, Gender and Season Are Good Predictors of Vitamin D Status Independent of Body Mass Index in Office Workers in a SubtropicalRegion." Nutrients 12.9 (2020): 2719.

[28] Al Zarooni, Amal Abdul Rahim, et al. "Prevalence of vitamin D deficiency and associated comorbidities among Abu Dhabi Emirates population." BMC research notes 12.1 (2019): 1-6.

[29] Mohn, Emily S., et al. "Evidence of drug-nutrient interactions with chronic use of commonly prescribed medications: An update." Pharmaceutics 10.1 (2018): 36.

[30] Vitamin D. Mayo Clinic. (2021). Retrieved 28 June 2021, from https://www.mayoclinic.org/drugs-supplements-vitamin-d/art20363792.

[31] Özdemir, Abdurrahman Avar, et al. "Vitamin D deficiency in pregnant women and their infants." Journal of clinical research in pediatric endocrinology 10.1 (2018): 44.

[32] Dharmshaktu, Pramila, et al. "Absence of vitamin D deficiency among common outdoor workers in Delhi." Clinical endocrinology 91.2 (2019): 356-362.

[33] O'sullivan, Fiona, et al. "Ambient UVB dose and sun enjoyment are important predictors of vitamin D status in an older population." The Journal of nutrition 147.5 (2017): 858-868.

[34] Jacob Schor, N. D. "Heliophobia: Fear of the Sun."

[35] Grant, William B., Harjit P. Bhattoa, and Pawel Pludowski. "Determinants of vitamin D deficiency from Sun exposure: a global perspective." Vitamin D. Academic Press, 2018. 79-90.

[36] Harkness, Laura S., and Barbara A. Cromer. "Vitamin D deficiency in adolescent females." Journal of adolescent health 37.1 (2005): 75.

[37] Mosekilde, Leif. "Vitamin D and the elderly." Clinical endocrinology 62.3 (2005): 265-281.

[38] Kweder, Hasan, and Housam Eidi. "Vitamin D deficiency in elderly: Risk factors and drugs impact on vitamin D status." Avicenna journal of medicine 8.4 (2018): 139.

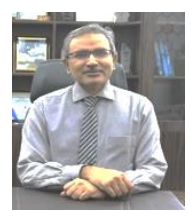

Dr. Muhammad Waris Farooka

Place \& date of birth: Lahore, Pakistan,1969.

FCPS (Pak), FRCS (Ed), FACS (USA), MCPS-HPE

(Pak).

Diploma Laparoscopic Surgery (France).

Member Society of American Gastrointestinal and Endoscopic Surgeons (SAGES).

Consultant Surgeon and Professor of Surgery.

Head of Surgery, Surgical ward II, Services Institute of Medical Sciences, Lahore, Pakistan.

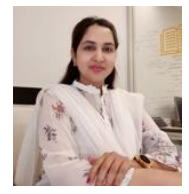

Dr. Syeda Saba Aslam

Place and date of birth: Karachi, Pakistan, 1981. MBBS (Dow, Pakistan), MPH (LSHTM, UK), IFOM (USA), CRCP (DUHS).

Head of Medical Affairs and Clinical Research, Scotmann Pharmaceuticals, Islamabad, Pakistan.

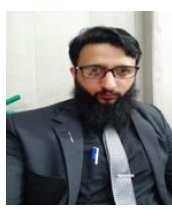

Dr. Mansoor Ahmad Tarar

Place \& date of birth: Mandi Bahauddin, Pakistan,1989 MBBS (Isra University), FCPS.

Resident General Surgery.

Clinical Registrar, Surgical Ward II, Services Institute

Medical Sciences, Lahore, Pakistan.

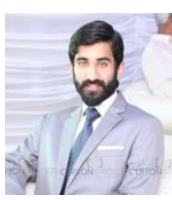

\section{Dr. Minhaaj Rafi}

Place and date of birth: Khanewal, Pakistan,1992.

MBBS, FCPS (General Surgery Trainee CPSP).

Academic Registrar.

Services Institute Medical Sciences, Lahore, Pakistan

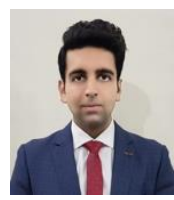

\section{Dr. Afaq Ahmad}

Place and date of birth: Khushab, Pakistan,1993.

MBBS (UHS), BSc (PU).

Post Graduate Resident Surgery.

Services Institute Medical Sciences, Lahore, Pakistan.

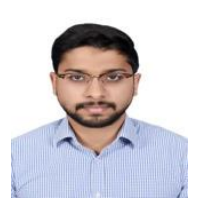

Dr. M Nouman Rafique

Place and date of birth: Lahore, Pakistan,1993.

MBBS, Post Graduate Resident General Surgery, Services Institute Medical Sciences, Lahore, Pakistan.

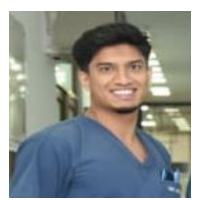

\section{Dr. Muhammad Bilal}

Place and date of birth: Lahore, Pakistan,1995.

MBBS, MS (General Surgery).

Services Institute of Medical Sciences, Lahore, Pakistan. 